\title{
LITERATUR
}

\section{Deutschland in Europa: Perspektiven zur Europäisierung deutscher Außenpolitik}

\author{
Lars Colschen*
}

Beiträge zur Europaforschung haben, nicht zuletzt im Zuge des gescheiterten Verfassungsvertrags und mit dem mühevollen Ringen um den Vertrag von Lissabon Hochkonjunktur. Der Bereich der deutschen Europapolitik ist trotz der hohen Verwobenheit von Außen- und Innenpolitik und den damit einhergehenden Schwierigkeiten einer strikten Begriffstrennung zwischen „Innen“ und „Außen“ analytisch dem Bereich der AuBenpolitik zuzuordnen. ${ }^{1}$ Das Buch „Deutsche Europapolitik. Von Adenauer bis Merkel“2 bildet den klassischen außenpolitischen Blickwinkel, in der Außenpolitik als Domäne der Regierung betrachtet wird, die gesellschaftliche Interessen zu wahren hat, ${ }^{3}$ in starkem Maße ab. Jenseits der Frage, ob und inwieweit eine nationale Europapolitik überhaupt noch autonom gestaltbar ist, ${ }^{4}$ wird die deutsche Europapolitik anhand von drei Leitfragen systematisch nachgezeichnet: Welchen Beitrag hat die Bundesrepublik Deutschland zur Vertiefung und zum Ausbau der EWG/ EG/EU geleistet? Inwiefern entspricht das deutsche europapolitische Engagement den nationalen Interessen? Inwieweit ist die grundsätzlich als europafreundlich einzustu-
Gisela Müller-Brandeck-Bocquet/Corina Schukraft/Nicole Leuchtweis/Ulrike Keßler: Deutsche Europapolitik. Von Adenauer bis Merkel, 2. aktualisierte und erweiterte Auflage, VS Verlag: Wiesbaden 2010, ISBN 978-3-531-16392-5; 349 Seiten, 24,90€.

Timm Beichelt: Deutschland und Europa. Die Europäisierung des politischen Systems, VS Verlag: Wiesbaden 2009, ISBN 978-3-531-15141-0; 364 Seiten, 29,90 €.

Dietrich von Kyaw: Auf der Suche nach Deutschland. Erlebnisse und Begegnungen eines deutschen Diplomaten und Europäers, Berliner Wissenschafts-Verlag: Berlin 2009, ISBN 978-38305-1634-7; 484 Seiten, 29,00 €.

fende deutsche Politik von Kontinuität und/ oder Wandel geprägt?

Das Ziel ist es, herauszuarbeiten, wie sich der Handlungs- und Gestaltungsspielraum Deutschlands im Prozess der europäischen Integration bisher entwickelt hat, welche Strukturmerkmale sich in der deutschen Europapolitik ausgeprägt haben und wie die heutige Rolle Deutschlands in der Europäischen Union zu bewerten ist.

* Dr. Lars Colschen, Geschäftsführer des Geschwister-Scholl-Instituts für Politikwissenschaft, Ludwig-Maximilians-Universität München.

1 Dazu siehe: Dirk Messner: Ist Außenpolitik noch Außenpolitik ... und was ist eigentlich Innenpolitik? Die Transformation der Politik in der „Ära des Globalismus“, in: Prokla. Zeitschrift für kritische Sozialwissenschaft 118/2000, S. 123-150.

2 Gisela Müller-Brandeck-Bocquet/Corina Schukraft/Nicole Leuchtweiß/Ulrike Keßler: Deutsche Europapolitik. Von Adenauer bis Merkel, 2. aktualisierte und erweiterte Auflage, Wiesbaden 2010.

3 Dazu siehe: Kai Oppermann/Alexander Höse: Die innenpolitischen Restriktionen deutscher Außenpolitik, in: Thomas Jäger/Alexander Höse/Kai Oppermann (Hrsg.): Deutsche Außenpolitik. Sicherheit, Wohlfahrt, Institutionen und Normen, Wiesbaden 2007, S. 40-68.

4 Dazu siehe: Timm Beichelt: Deutschland und Europa. Die Europäisierung des politischen Systems, Wiesbaden 2009. 
In diesem Sinne zeichnen die vier Autorinnen die deutsche Europapolitik in insgesamt fünf Beiträgen seit Gründung der Bundesrepublik entlang der verschiedenen Regierungen historisch-empirisch nach.

Corina Schukraft beschäftigt sich zum Auftakt mit den Regierungen unter den Kanzlern Adenauer, Erhard und Kiesinger und damit mit den Anfängen der deutschen Europapolitik in den 1950er und 1960er Jahren. Dabei werden alle maßgeblichen erfolgreichen (zum Beispiel die Römischen Verträge und der deutsch-französische Freundschaftsvertrag) wie gescheiterten (zum Beispiel die Gründung der Europäischen Verteidigungsgemeinschaft (EVG) und die Fouchet-Pläne) europapolitischen Vorhaben beleuchtet. Am Schluss jeder Betrachtung steht eine knappe Bilanz. Dabei stellt die Verfasserin fest, dass - im Unterschied zur Adenauer-Ära - den Regierungen Erhard und Kiesinger die großen europapolitischen Erfolge verwehrt blieben. Dies war in erster Linie dem Umstand geschuldet, dass beide Regierungen es nicht verstanden, die unterschiedlichen Interessen Deutschlands und Frankreichs miteinander zu verbinden. Gerade dies aber war Adenauer meisterhaft gelungen. ${ }^{5}$

Die SPD-Kanzlerschaften von Willy Brandt und Helmut Schmidt werden von Nicole Leuchtweis aufgearbeitet. Auch hier werden alle zentralen Wegmarken der deutschen Europapolitik (zum Beispiel die Begründung der Europäischen Politischen Zusammenarbeit, EPZ) nachgezeichnet. Bei der Regierung Willy Brandt wird die Neue Ostpolitik leider stiefmütterlich behandelt. Sie gehört zwar nicht zur deutschen (West-)Europapolitik in einem engeren, institutionenbezogenen (EG-) Sinne. Aber dieser Meilenstein bundesdeutscher Außenpolitik hätte wegen seiner Bedeu- tung für den europäischen Einigungsprozess durchaus eine gewichtigere Berücksichtigung verdient. Grundsätzlich muss der Leser aber dankbar sein, dass die in vielen Darstellungen zur deutschen Europapolitik meist unterbelichtete EG-Politik der Regierung Brandt hier kenntnisreich herausgearbeitet worden ist.

Die Außenpolitik der Regierung Kohl, bei deren Darstellung die EG-/EU-Politik meist ohnehin eine dominante Rolle einnimmt, ist Gegenstand des Beitrags von Ulrike Keßler. Hier geht es unter anderem um die Überwindung der ersten Eurosklerose und die Reanimation des europäischen Einigungsprozesses in den 1980er Jahren. Breiten Raum nehmen auch der bahnbrechende Vertrag von Maastricht, die Wirtschafts- und Währungsunion, die Gemeinsame Außen- und Sicherheitspolitik (GASP) und die Vorbereitungen der späteren Osterweiterung der Europäischen Union ein.

Gisela Müller-Brandeck-Bocquet nähert sich mit ihren beiden Beiträgen über die rot-grüne Europapolitik und die EU-Politik unter der Kanzlerschaft von Angela Merkel dann sukzessive an die Gegenwart an. Im Brennpunkt der Ära Schröder/Fischer stehen unter anderem die deutsche Ratspräsidentschaft 1999, der Krisengipfel von Nizza 2000 und der gescheiterte EU-Verfassungsprozess. Etwas schmal fällt die Abhandlung über die vielbeachtete Humboldt-Rede von Joschka Fischer vom 12 . Mai $2000^{6}$ aus, die sogenannte Finalitätsdebatte europaweit ausgelöst hatte.

Die Ära Merkel ist unter anderem gekennzeichnet durch die deutsche Ratspräsidentschaft 2007, die Themen Klimaschutz und Energiesicherheit sowie die Erweiterungspolitik und die Bemühungen um die Stärkung der GASP. Die (vorläufige) Bewertung der europapolitischen Bilanz der Kanzlerschaft

5 Corina Schuhkraft: Die Anfänge deutscher Europapolitik in den 50er und 60er Jahren, in: Gisela Müller-Brandeck-Bocquet/Corina Schukraft/Nicole Leuchtweiß/Ulrike Keßler: Deutsche Europapolitik, 2010, S. 13-66, hier S. 47.

6 Joschka Fischer: Vom Staatenverbund zur Föderation - Gedanken über die Finalität der europäischen Integration, Vortrag an der Humboldt-Universität zu Berlin am 12. Mai 2000, Forum Constitutionis Europae: FCE 12/00 (Spezial 2). 
von Angela Merkel, fällt zwar etwas milder aus, als bei der rot-grünen Vorgängerregierung, der „der große Erfolg versagt blieb“.7 Dennoch wird auch dem Tandem Merkel/ Steinmeier „Visionslosigkeit“ attestiert. ${ }^{8}$ Aufgrund des Erscheinungsdatums des Buches kann sich die Analyse der deutschen Europapolitik unter Merkel nur auf die schwarz-rote (,große') Koalition beziehen. Dennoch wird das Inkrafttreten des Vertrags von Lissabon bereits antizipiert. Insofern wäre zumindest ein Ausblick auf Perspektiven oder Szenarien für die deutsche Europapolitik im post-Lissabon-Prozess zur Abrundung des Beitrags durchaus lohnenswert gewesen.

„Deutsche Europapolitik. Von Adenauer bis Merkel" ist nach 2002 die zweite Auflage dieses Buches. Sie bietet sich aufgrund ihrer gestrafften Darstellung als hervorragender Einstieg und Grundlage für die Beschäftigung mit dieser Thematik an. Obwohl es sich um vier verschiedene Autorinnen handelt, hat der Leser den Eindruck, dass die fünf Kapitel aus einem Guss geschrieben sind, denn sie sind ähnlich strukturiert und gehen nahezu nahtlos ineinander über. Leider sind die ersten drei Beiträge und damit die deutsche Europapolitik von 1949 bis 1998 in der zweiten Auflage keiner Überarbeitung unterzogen worden. Das ist zumindest schade, da Forschung und $\mathrm{Pu}$ blikationstätigkeit zur deutschen Europapolitik in den vergangenen Jahren auch zum Zeitraum 1949 bis 1998 vorangeschritten sind. ${ }^{9}$

Der europäische Integrationsprozess hat die politischen Prozesse und Institutionen der Bundesrepublik nachhaltig verändert. Dennoch wird in der Europaforschung die Europäische Union oft nicht als systemische Komponente deutscher Politik begriffen. Statt die europäische und die nationale Ebene mit allen ihren Aus- und Rückwirkungen systematisch miteinander zu verknüpfen, wird häufig von einer autonom gestaltbaren Europapolitik ausgegangen. Dies sei, so der Autor, zu kurz gegriffen, zumal nationale Akteure bereits im Vorfeld von in Brüssel getroffenen Entscheidungen an deren Formulierung beteiligt sind. Ferner richten sich die Handlungserwartungen nationaler Akteure nicht (mehr) nur punktuell, sondern systematisch an europäische Institutionen, da ein hoher Anteil deutscher Rechtsakte über die EU-Ebene eingespeist wird.

Hier setzt der Beitrag an, den Timm Beichelt zur Europaforschung leisten möchte. ${ }^{10} \mathrm{Er}$ geht der Leitfrage nach: Wie ist es zu erklären, dass der in Deutschland dominante integrationsbefürwortende Konsens zugunsten einer distanzierteren Europaeinstellung abgebröckelt ist?

Dabei ist es nicht sein Ziel, eine Großtheorie zur Europäisierung des deutschen Regierungssystems zu entfalten, aber er erhebt den Anspruch, ein ,integriertes Analysekonzept zur Bestimmung der transnationalen Verwobenheit deutscher Politik zu entwickeln." ${ }^{11}$ Das variantenreiche Europäisierungskonzept wird dabei nicht nur als eine gleichartig verlaufende Anpassungsdynamik des nationalen Systems an die europäische Ebene begriffen. Vielmehr sind einzelnen nationalen Institutionen unterschiedliche Europäisierungsmodelle zuzuordnen. Dieser Ansatz steht damit durchaus im Kontrast zu der lange Zeit in der Europaforschung dominierenden Ausrichtung, die Europapolitik lediglich als ein außenpolitisches Themenfeld neben anderen begreift.

7 Gisela Müller-Brandeck-Bocquet: Rot-grüne Europapolitik 1998-2005: Eine Investition in die Zukunft der EU, in: Gisela Müller-Brandeck-Bocquet/Corina Schukraft/Nicole Leuchtweiß/Ulrike Keßler: Deutsche Europapolitik, 2010, S. 173-252, hier S. 245.

8 Ebenda, S. 342.

9 Dazu zum Beispiel Henning Türk: Die Europapolitik der Grossen Koalition 1966-1969, München 2006 oder Henrik Meyer: Deutsche Europapolitik unter Helmut Kohl - die Auswirkungen des politischen Umfeldes auf die Integrationsbereitschaft der Bundesregierung, Berlin 2004.

10 Beichelt: Deutschland und Europa, 2009.

11 Ebenda, S. 13. 
Der Autor nähert sich seinem Thema, in dem er zunächst einmal den deutschen politischen Prozess in die Prozessabläufe des Mehrebenensystems, den europäischen Politik-Zyklus systematisch einordnet. In einem nächsten Schritt werden die Einstellungen der Deutschen zu Europa beleuchtet. Der Befund lautet, dass die europäische Identitätsdimension deutlich zugenommen hat. Sie ist additiv neben das Nationale getreten, ersetzt es aber nicht. Die jeweilige Ausprägung einer europäischen Identität variiert dabei von Politikfeld zu Politikfeld. Ferner stellt Beichelt fest, dass gar kein grundsätzlicher Rückgang der Hinwendung zu Europa stattgefunden hat, sondern die distanziertere Europahaltung (das war die zu erklärende Ausgangsbeobachtung) sich primär auf die Praxis des europäischen Regierens und die europäischen Institutionen bezieht. Nach dieser wenig überraschenden Erkenntnis wird detailliert und kenntnisreich aufgezeigt, wie eine deutsche Bundesregierung ,,in einem verzweigten Geflecht politisch-ideologischer, gesellschaftlicher und regionaler Interessen agiert, das den nationalen Horizont übersteigt."12 Ein Ergebnis dieser empirisch-analytischen Untersuchung ist, dass eine Akzentverschiebung vom autonomen zum vernetzten Akteur aufgetreten ist, die ,deutlich Zweifel an der Homogenitätsannahme des deutschen Regierungshandelns aufkommen" lässt. ${ }^{13}$

Im Schlusskapitel widmet sich Beichelt der Frage, ob und inwieweit die festgestellten Europäisierungstendenzen zu einer ,substantiellen Beschädigung" des politischen Systems der Bundesrepublik geführt haben. ${ }^{14}$ Diese Fragestellung impliziert zunächst einmal, dass im deutschen Fall keine positiven Demokratisierungseffekte des nationalen Systems durch die Europäische Union stattgefunden haben, wie es zum Beispiel für mittel- und osteuropäische Staaten anzunehmen ist, sondern wenn, dann eher gegenläufige Tendenzen feststellbar sind. Aber im Ergebnis war es dem Autor letztlich ,nicht möglich, zu einer eindeutigen Bilanz zu gelangen." ${ }^{15}$ Er spricht daher von einer Charakterveränderung der deutschen Demokratie durch ,eine beträchtliche Umorientierung auch des nationalen Institutionensystems", ${ }^{16}$ bei der sich das Verhältnis von Regierenden und Regierten von einer partizipativen zu einer responsiven Dimension verschoben hat. ${ }^{17}$ Dabei enthält er sich einer Einschätzung, ob und inwieweit das nun letztlich als substanzieller Schaden für die deutsche Demokratie zu bewerten ist.

Insgesamt steht die Arbeit nicht in der Kontinuitätslinie der Mehrzahl der Arbeiten in der Europaforschung. Sie hat daher das Verdienst, einen noch jungen Diskussionsraum, der die Facette der Europäisierung des politischen Systems der Bundesrepublik stärker und systematischer in den Fokus der wissenschaftlichen Betrachtung rückt, weiter geöffnet zu haben.

Dietrich von Kyaw war als deutscher Diplomat seit 1964 in verschiedenen Funktionen unter anderem an den Botschaften im Kongo, in der Zentralafrikanischen Republik und in Washington D.C. tätig. Darüber hinaus vertrat er die Bundesrepublik bei den Vereinten Nationen in New York und war am Ende seiner diplomatischen Laufbahn von 1993 bis 1999 der Ständige Vertreter der Bundesrepublik Deutschland bei der Europäischen Union.

Von Kyaw blickt auf eine lange und ereignisreiche Diplomatenlaufbahn zurück, ${ }^{18}$ die er nach dem Studium der Politik- und Rechts-

12 Ebenda, S. 207.

13 Ebenda.

14 Ebenda, S. 328.

15 Ebenda.

16 Ebenda, S. 330.

17 Ebenda, S. 16

18 Dietrich von Kyaw: Auf der Suche nach Deutschland. Erlebnisse und Begegnungen eines deutschen Diplomaten und Europäers, Berlin 2009. 
wissenschaften an den Universitäten Bonn, Chicago und Lüttich im Jahre 1964 begonnen hatte. Er erlebte dabei hautnah deutsche Geschichte von einer enormen Spannbreite. Seine Laufbahn begann im deutschen Generalkonsulat in Los Angeles, wo er bei KZProzessen mit der deutschen Vergangenheit konfrontiert wurde und endete als Vertreter des vereinten Deutschlands bei einer (in Teilen) supranationalen Organisation. Dazwischen erlebte er in der Zentralafrikanischen Republik den Diktator Bokassa und in New York 1973 den Beitritt beider deutscher Staaten zu den Vereinten Nationen.

Den weitaus größten Raum - mit rund 150 Seiten etwa ein Drittel des Buches - nehmen die sechs Jahre als Ständiger Vertreter der Bundesrepublik Deutschland bei der Europäischen Union ein. Von Kyaw bezeichnet dies als seinen Traumposten, da er als überzeugter Europäer den europäischen Einigungsprozess direkt vor Ort mitgestalten konnte. ${ }^{19}$ Einen besonderen Einblick in die Geheimnisse multilateraler Aushandlungsprozesse gewährt das Kapitel über die deutsche EU-Ratspräsidentschaft 1999. Wenn seine Bilanz der Ratspräsidentschaft aus deutscher Sicht an sich durchaus positiv ausfällt, so sei dies weniger dem Duo Schröder/Fischer zuzurechnen, als vielmehr ,einer europapolitisch hinreichend solide ausgerichteten Beamtenschaft. "20 Ohnehin lässt die Schärfe, mit der der Autor die Europapolitik der rot-grünen Bundesregierung kritisiert, wenig zu wünschen übrig, wenn er zum Beispiel feststellt: „Weil Schröder insofern kein Kohl war, verspielte Deutschland unter ihm weitgehend seine in der EU unersetzbare Mittlerrolle. Es reicht nicht mehr, nur im Gleichschritt mit Frankreich zu agieren. Erst Angela Merkel gelang es, Deutschland wieder zum ehrlichen Makler in der EU zu machen.“21

Wenn auch das biografische Moment dominiert, so ist das Buch dennoch als Mischung aus Biografie und Sachbuch zu charakterisieren. Von Kyaw zeigt sich als scharfer Beobachter, der Situationen zu analysieren versteht und entsprechende Schlussfolgerungen für die deutsche Außenpolitik zieht. Diese fallen auch jenseits der Bewertung der Außenpolitik der rot-grünen Bundesregierung nicht selten durchaus kritisch aus. So sprach er sich gegen die „zu einseitige Vorliebe“ von Außenminister Genscher für die Entspannungspolitik unter Vernachlässigung der Realpolitik aus. ${ }^{22}$ Von Kyaw garniert seine Ausführungen durch zahlreiche Anekdoten und persönliche Erfahrungen, die das Buch überaus lesenswert machen. Daher wäre es auch verfehlt, strenge wissenschaftliche Maßstäbe anzulegen. Dennoch eröffnen sich auch aus wissenschaftlicher Perspektive interessante Einblicke in Fragen wie zum Beispiel zu den Funktionsbedingungen und Handlungsspielräumen von Diplomatie.

Dieses Buch kann all denen wärmstens ans Herz gelegt werden, die die Praxis der deutschen Außenpolitik einmal durch die Brille eines Diplomaten betrachten wollen oder die berufsperspektivisch mit einer Laufbahn im Auswärtigen Amt liebäugeln (auch wenn sich das Berufsbild Diplomat mittlerweile weiter gewandelt hat). 